\title{
Optimization of aviation adjuvants based on wettability analysis for insecticide application on maize using UAV
}

\author{
Yu Zang ${ }^{1,2,3}$, Zhiyan Zhou ${ }^{1,2,3}$, Ying Zang ${ }^{1,2,3^{*}}$, Xiwen Luo ${ }^{1,3}$, Juan Liao ${ }^{1,3}$, Rui Ming ${ }^{1,2,3}$, \\ Cancan Song ${ }^{1,2,3}$, Le Zi ${ }^{1,2,3}$, Rui Jiang ${ }^{1,2,3}$, Hanxiang Xiao ${ }^{4}$ \\ (1. College of Engineering, South China Agricultural University / Guangdong Engineering Research Center for Agricultural Aviation \\ Application (ERCAAA), Guangzhou 510642, China; \\ 2. National Center for International Collaboration Research on Precision Agricultural Aviation Pesticides Spraying Technology (NPAAC), \\ Guangzhou 510642, China; \\ 3. Key Laboratory of Key Technology on Agricultural Machine and Equipment (South China Agricultural University), \\ Ministry of Education, Guangzhou 510642, China; \\ 4. Institute of Plant Protection, Guangdong Academy of Agricultural Sciences, Guangzhou 510640, China)
}

\begin{abstract}
Adjuvants can increase the control efficacy of diseases and insect pests by changing the physico-chemical properties of pesticides. Most of the aviation spray adjuvants are versions of ground adjuvants. Maize insecticide sprays with unmanned aerial vehicles (UAV), have problems such as relatively low droplet deposition rate and poor wettability. Hence, wettability research and optimization tests for aviation spray adjuvants are needed. The present study screened 12 spray adjuvants using physico-chemical property experiments. The adjuvants were applied to improve the droplet deposition and control efficacy in maize borers controlling by UAV. The selected spray adjuvants were Po2 (a hyperbranched polymer adjuvant) and VO3 (a vegetable oil adjuvant). Results showed that, (1) When Po2 was added in water-dispersible granules (WGs) at volume rate of $12 \mathrm{~L} / \mathrm{hm}^{2}$, the dynamic surface tension (DST) of WGs was decreased by $37.41 \%$, and the maize leaves were covered by droplets $100 \%$, the droplet deposition was increased by $104 \%$ and maize borer control was increased by $46 \%$; (2) VO3 decreased the surface tension of ultra-low-volume (ULV) formulation by $12.02 \%$ and the maize leaves were covered by droplets $100 \%$. The effect of VO3 on improving the droplet deposition and control efficacy at $12 \mathrm{~L} / \mathrm{hm}^{2}$ with ULV was not significant. Thus, the addition of aviation spray adjuvant to improve the wettability of WGs significantly improved the droplet deposition and control efficacy but it had no significant effect on the ULV formulation.
\end{abstract}

Keywords: unmanned aerial vehicles, spray adjuvants, wettability, maize insecticide application

DOI: $10.25165 /$ j.ijabe.20211405.6605

Citation: Zang Y, Zhou Z Y, Zang Y, Luo X Y, Liao J, Ming R, et al. Optimization of aviation adjuvants based on wettability analysis for insecticide application on maize using UAV. Int J Agric \& Biol Eng, 2021; 14(5): 11-18.

\section{Introduction}

Maize is one of the most widely distributed food crops in the world $^{[1]}$. However, maize productivity is affected by biological factors such as pests and diseases. The application of chemicals remains the major tactic for pest and disease control ${ }^{[2]}$. High planting densities and high crop plants limit the ability of traditional sprayers for chemical sprays in maize ${ }^{[3]}$. Unmanned

Received date: 2021-03-22 Accepted date: 2021-07-30

Biographies: Yu Zang, PhD, research interests: agricultural aviation application, Email: zangyu@scau.edu.cn; Zhiyan Zhou, PhD, Professor, research interests: agricultural aviation application, Email: zyzhou@scau.edu.cn; Xiwen Luo, Professor, research interests: precision agriculture, Email: xwluo@scau.edu.cn; Juan Liao, $\mathrm{PhD}$, research interests: agricultural aviation application, Email: liaojuan0529@126.com; Rui Ming, PhD, research interests: formation control and neural network control, Email: elcondorpasa502100347@stu.scau.edu.cn; Cancan Song, $\mathrm{PhD}$, research interests: variable-rate technology, Email: songcc@stu.scau.edu.cn; Le Zi, MD, research interests: agricultural aviation application, Email: 891750786@qq.com; Rui Jiang, PhD, research interests: agricultural aviation application, Email: 469289264@qq.com; Hanxiang Xiao, Professor, research interests: occurrence regularity and integrated control technique of pests. Email: 290432210@qq.com.

*Corresponding author: Ying Zang, $\mathrm{PhD}$, Professor, research interests: agricultural mechanization and automation, College of Engineering, South China Agricultural University, Guangzhou 510642, China. Tel: +86-13724090346, Email: yingzang@scau.edu.cn. aerial vehicles (UAVs) were proved as efficient sprayers of plant protection chemicals ${ }^{[4]}$. Compare with other sprayers, UAVs can reduce pesticide $\operatorname{losses}^{[5]}$ and minimize applicator exposure ${ }^{[6]}$ to chemical. UAVs are valuable for treating complex terrain and small farms with separate plots and they do not require a specific takeoff airport ${ }^{[7]}$. UAVs are easy to be operated and with good adaptive capacity in spraying environment ${ }^{[8]}$. They have great potential for maize pest and disease control treatments in China.

During UAV application flights, aerial spray droplets are affected by the rotor wind field and natural wind. This results in uneven deposition of the droplets, which are prone to drift and rolling ${ }^{[9]}$. Uneven deposition leads to the spray not providing adequate coverage ${ }^{[10]}$. Spray adjuvants are chemicals added to improve the physical properties and effectiveness of pesticide liquids. They can decrease the surface tension of droplets, modify the droplet size distribution, increase the retention of liquid on the leaves, and improve the utilization rate of the active ingredient ${ }^{[11,12]}$ Aviation spray adjuvants can play an important role in the use of UAV for plant protection.

Most studies on the use of UAV in maize have focused on remote sensing, such as estimation of maize biomass ${ }^{[13]}$, evaluation of nitrogen effects ${ }^{[14]}$, and water stress monitoring ${ }^{[15]}$. Xiao et al. ${ }^{[16]}$ found that a vegetable oil adjuvant significantly increased the droplet coverage rate and the retention of defoliants on cotton leaves. However, few studies have addressed adjuvants in 
formulations applied to maize. Meng et al. ${ }^{[17]}$ found that adjuvants can improve the efficiency of UAV sprays and reduce the dosage of the insecticide imidacloprid by $20 \%$. These results indicate that adjuvants used with formulations applied by UAV can have a positive effect.

However, aviation spray adjuvants can have variable effects combined with different pesticides due to differences in their physico-chemical properties. The adjuvants that are suitable for different pesticides may also differ ${ }^{[18,19]}$. The wetting behavior of pesticide spray liquid on plant surfaces is important for spray applications. Differences in wettability of pesticide can change the spray wetting behavior ${ }^{[20,21]}$. Greater wettability of the pesticide results in less rolling loss and increased droplet deposition. There is limited information on the relationship between the wettability of aviation spray adjuvants and spray quality and efficacy.

The objective of this study was to improve the spray effect and control efficacy in insecticide application on maize using UAV.
12 kinds of aviation spray adjuvants were optimized by dynamic surface tension (DST) and contact angle (CA) analysis in the laboratory. And then the two kinds of optimum adjuvants were used in maize insecticide spray formulations to improve spray effect and control efficacy in field study.

\section{Materials and methods}

\subsection{Wettability properties experiment}

Decrease the DST and CA can improve the wetting deposition of the liquid on the target surface, improve the penetration and transmission of active ingredients, and improve the control efficacy $^{[22]}$. Therefore, the DST and CA values on maize leaves were used to select adjuvants to improve the efficacy of field experiments.

2.1.1 Pesticides and aviation spray adjuvants for test

Many kinds of spray adjuvants are available in China. Table 1 lists 12 kinds of the most common spray adjuvants selected for evaluation.

Table 1 Aviation spray adjuvants tested

\begin{tabular}{clll}
\hline Serial No. & \multicolumn{1}{c}{ Product name } & \multicolumn{1}{c}{ Components } \\
\hline VO1 & Beidatong & Methylate vegetable oil & Manufacturer \\
VO2 & Plant essential oil & Vegetable oil & Qingdao Lvlong Crop nutrition Co., Ltd. \\
VO3 & Aero-mate 320 & Vegetable oil & Solvay (Zhenjiang) Chemicals Co., Ltd. \\
VO4 & Nongboshi & Vegetable oil & Guangxi Tianyuan Co., Ltd. \\
VO5 & TIS-331 & Vegetable oil & Jiangxi Tiansheng New Material Co., Ltd \\
Si1 & Qigong & silicone & Guilin Jiqi Group Co., Ltd. \\
Si2 & Quanfeng & silicone & Anyang Quanfeng Biological Technology Co., Ltd. \\
Po1 & Beibeija & Aliphatic amine polymers & Guilin Jiqi Group Co., Ltd. \\
Po2 & Nongjianfei & hyperbranched polymer & Guilin Jiqi Group Co., Ltd. \\
Mix1 & Feishoubao & A mixture of vegetable oils and polymers & Nantong Pest Agrochemical Co., Ltd. \\
Mix2 & Hongyuyan & A mixture of condensation compound, & Shenzhen Noposion International Investment Co., Ltd. \\
Le1 & Kesheng & alcohol ethoxylate and polyol & Jiangsu Kesheng Group Co., Ltd. \\
\hline
\end{tabular}

One tested pesticide was Virtako $40 \mathrm{WG}$, a water-dispersible granule (WG) produced by Syngenta Crop Protection Co., Ltd. with the active ingredients chlorantraniliprole and thiamethoxam. The other tested pesticide was an ultra-low-volume (ULV) formulation produced by Guizhou University. The active ingredients of this ULV formulation were $1 \%$ chlorantraniliprole and $1 \%$ thiamethoxam. The organic solvent is 5\% NMP (N-Methyl pyrrolidone) and 8\% Cyc (Cyclohexanone). The rest of the ULV is made up of $35 \%$ methyl oleate and chlorinated paraffin. Different aviation spray adjuvants were added to the pesticides to make $1 \%$ solutions for evaluation.

\subsubsection{Dynamic surface tension measurements}

The dynamic surface tension (DST) of the test solution was measured with a surface tensiometer (EZ-Pi) (Kibron Inc. Oy., Helsinki, Finland). Before each measurement, distilled water was used to calibrate the instrument. The device uses the Du-Nouy method to measure surface tension with a measurement range of $1-300 \mathrm{mN} / \mathrm{m}$ and an accuracy of $0.1 \mathrm{mN} / \mathrm{m}$. The average value of three measurements was taken as the surface tension of the solution.

\subsubsection{Contact angle measurements}

The contact angle (CA) of the solution was measured with a PT-705-B (Precise Test, Guangdong, China). Healthy and intact maize blades were selected for the test. The blade surface dust was removed with clean water and the blades were clamped tightly to avoid their influence on the test results. The CA of $3 \mu \mathrm{L}$ droplets on maize leaves at $0 \mathrm{~s}, 10 \mathrm{~s}, 30 \mathrm{~s}, 60 \mathrm{~s}, 90 \mathrm{~s}$, and $120 \mathrm{~s}$ were recorded by the contact angle meter (Figure 1). According to the actual situation of CA, an elliptical fitting method or a circular fitting method was selected to measure the CA of the sample.

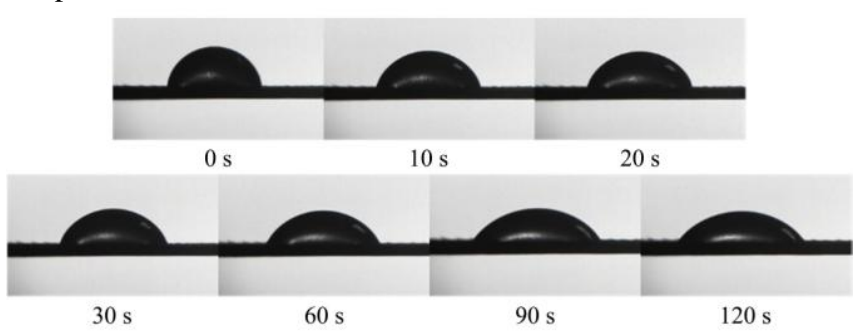

Figure 1 Process of droplet spreading on a maize leaf

\subsection{Field experiment}

Based on the screening results of aviation spray adjuvant for two pesticides (WG and ULV), field experiments of UAV spraying maize insecticide were conducted to compare the control efficacy of the two pesticide dosage forms and verify the effect of the aviation spray adjuvant, and compare the spray effect of UAV with two other types of sprayers.

\subsubsection{Field plots}

The field study was carried out at Zengcheng Teaching and Research Bases (11338' 38.0' ' E, 23ำ $14^{\prime} 34.7^{\prime}$ ' N), South China Agricultural University, Guangzhou, China, from June 27 to July 4 in 2018. The maize variety tested was No. 8 Huameitian, 
which is one of the main maize varieties in South China. The average plant height of the maize was $1.68 \mathrm{~m}$ at the heading stage. The major insect pests in the field were second and third instar Asian maize borers.

\subsubsection{UAV and ground sprayers}

A four-rotor electric plant protection UAV P20 (Xaircraft

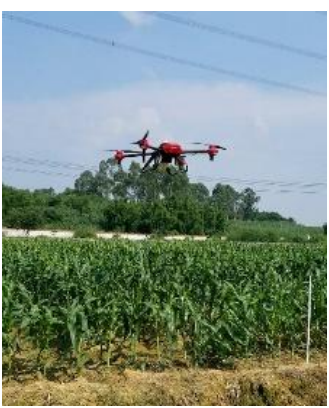

a. UAV

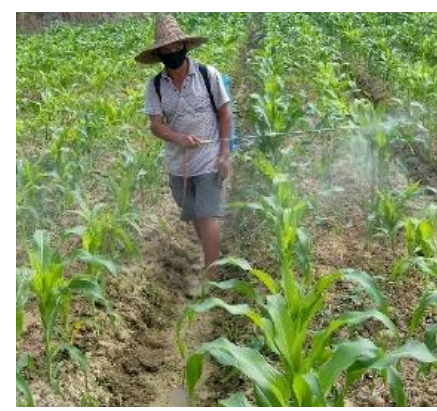

b. EAP sprayer
Technology Co., Ltd., Guangzhou, China) was used in this study; a high ground-clearance (HGC) sprayer 3WPZ-700 (Qingzhou Jiayi Agricultural Equipment Co., Ltd, Qingzhou, China) and an EAP sprayer 3WBD-18 (Guangzhou Nongyou Farm Tools Products Co., Ltd. Guangzhou, China) were used as references (Figure 2). The specifications of three sprayers are shown in Table 2.

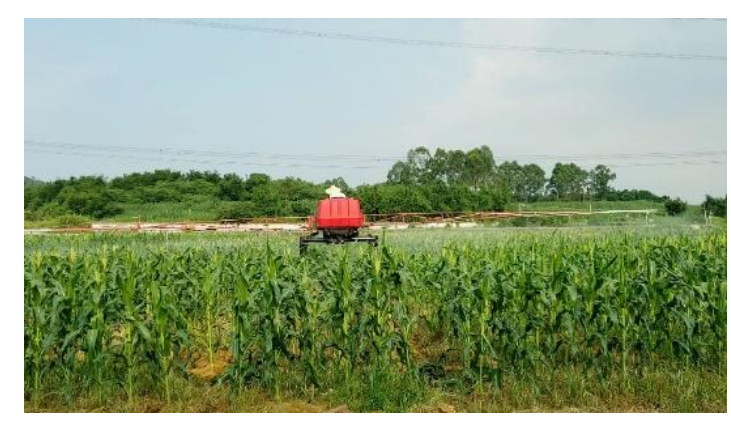

c. HGC

Figure 2 UAV and reference sprayers

Table 2 Specifications of the three research sprayers

\begin{tabular}{lccc}
\hline \multicolumn{1}{c}{ Parameters } & P20 & 3WPZ-700 & 3 WBD-18 \\
\hline Size/mm & $1180 \times 1180 \times 410$ & $4510 \times 1840 \times 2680$ & $370 \times 195 \times 478$ \\
Payload/L & 10 & 700 & 16 \\
Nozzle type & 4 centrifugal nozzles & 21 flat-fan nozzles & 1 flat-fan nozzle \\
Flow rate/L·min ${ }^{-1}$ & $3.6-5.6$ & $16.8-42$ & $1.2-1.8$ \\
Spray pressure/MPa & $/$ & $0.3-0.5$ & $0.18-0.42$ \\
Spray swath width/m & 3.5 & 12 & $/$ \\
Ground clearance/m & $/$ & 1.7 & $/$ \\
Tread/mm & $/$ & 1740 & $/$ \\
\hline
\end{tabular}

\subsubsection{Test scheme}

There were nine treatments, including six treatments using
UAV, one treatment using the high ground-clearance sprayer, one treatment using the EAP sprayer, and one blank treatment. Each UAV test plot was $100 \mathrm{~m}$ long and $10.5 \mathrm{~m}$ wide (three UAVs' spray swath widths), with three variables: pesticide formulation (WG, ULV), application rate $\left(12 \mathrm{~L} / \mathrm{hm}^{2}, 15 \mathrm{~L} / \mathrm{hm}^{2}\right)$, and screened spray adjuvant $(0,1 \%)$. The test plot for the high ground-clearance sprayer was $100 \mathrm{~m}$ long and $24 \mathrm{~m}$ wide (two spray swath widths). Test plots for the EAP sprayer and blank treatment were the same size as the UAV treatment. The test scheme is listed in Table 3. The climatic conditions were recorded using a weather meter, which indicated temperatures of $28.6^{\circ} \mathrm{C}-33.6^{\circ} \mathrm{C}$, relative humidity of $60.1 \%-68.5 \%$, and wind velocities of $0.0-0.8 \mathrm{~m} / \mathrm{s}$.

Table 3 Test scheme

\begin{tabular}{|c|c|c|c|c|c|c|c|}
\hline Test plot & $\begin{array}{l}\text { Pesticide } \\
\text { formulation }\end{array}$ & $\begin{array}{l}\text { Application rate } \\
/ \mathrm{L} \cdot \mathrm{hm}^{-2}\end{array}$ & $\begin{array}{l}\text { Spray adjuvant } \\
\quad / \mathrm{mL} \cdot \mathrm{L}^{-1}\end{array}$ & $\begin{array}{l}\text { Chlorantraniliprole } \\
\qquad \mathrm{g} \cdot \mathrm{L}^{-1}\end{array}$ & $\begin{array}{c}\text { Thiamethoxam } \\
/ \mathrm{g} \cdot \mathrm{L}^{-1}\end{array}$ & Applied rate & Sprayers \\
\hline A1 & WG & 12 & I & 2.5 & 2.5 & \multirow{8}{*}{30 g.a.i $\cdot \mathrm{hm}^{-2}+30$ g.a.i $\cdot \mathrm{hm}^{-2}$} & \multirow{6}{*}{ UAV } \\
\hline $\mathrm{A} 2$ & WG & 12 & 10 & 2.5 & 2.5 & & \\
\hline A3 & WG & 15 & I & 2 & 2 & & \\
\hline A4 & ULV & 12 & I & 2.5 & 2.5 & & \\
\hline A5 & ULV & 12 & 10 & 2.5 & 2.5 & & \\
\hline A6 & ULV & 15 & I & 2 & 2 & & \\
\hline B & WG & 900 & l & 0.033 & 0.033 & & HGC sprayer \\
\hline $\mathrm{C}$ & WG & 450 & l & 0.066 & 0.066 & & EAP sprayer \\
\hline $\mathrm{CK}$ & I & I & I & & & & I \\
\hline
\end{tabular}

\subsubsection{Characterization of spray deposition}

The eight treatments (six with the UAV, one with the EAP, and one with the HCG) described above were applied on June 27, 2018. Deposition under the sprayers varied across the swath and along the spray path with changing meteorological conditions and operation. For this reason, sample locations in this study were selected to account for this variation, as far as possible, and an optical method was used for modification according to Kirk et $\mathrm{al}^{[23]}$. Prior to the spray application in each treatment, artificial samplers were placed in ten equally spaced sample sites across the center of the sprayer (Figure 3). Each sample site was $0.5 \mathrm{~m}$ apart and spanned a total of $4.5 \mathrm{~m}$. The sample sites were repeated three times along the spray direction, with an interval of $20 \mathrm{~m}$ between each repetition. To avoid cross contamination between plots, sampling was performed at the center of each plot (Figure 3).
The artificial samplers at each sample site consisted of one water sensitive papers (WSPs) or oil sensitive papers (OSPs) $(25 \mathrm{~mm} \times 75 \mathrm{~mm}$ ) (Figure 4). The objectives of the WSPs or OSPs were to assess droplet parameters, including the area of coverage, droplet size and number of spray deposits at different heights. After each treatment replication, sufficient time was allowed for the droplets deposited on the WSPs and OSPs ( $\sim 5 \mathrm{~min})$. All samples were quickly removed and placed in zip-lock bags along with a label describing the treatment, replication, and sample site information, and then transported to a drying oven. This was done to prevent the WSPs from becoming moist on the samplers.

WSPs and OSPs were scanned at a resolution of 600 dots per inch (DPI) with a scanner, and imagery software DropletScan (USDA, USA) ${ }^{[24]}$ was utilized to extract and analyze the stain diameter, area of coverage, droplet deposition, and number of spray deposits on the WSPs ${ }^{[25]}$. 


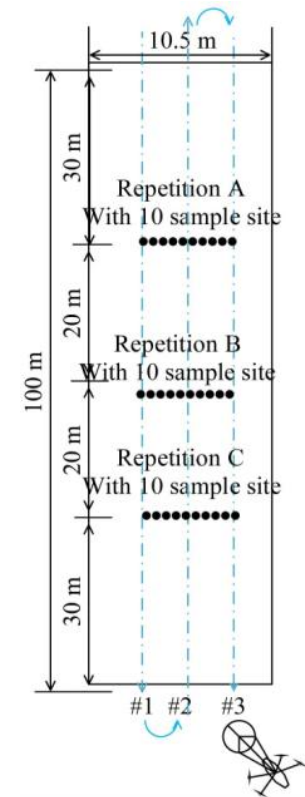

Figure 3 Experimental layout of each plot in relation to the unmanned aerial vehicle sprayer
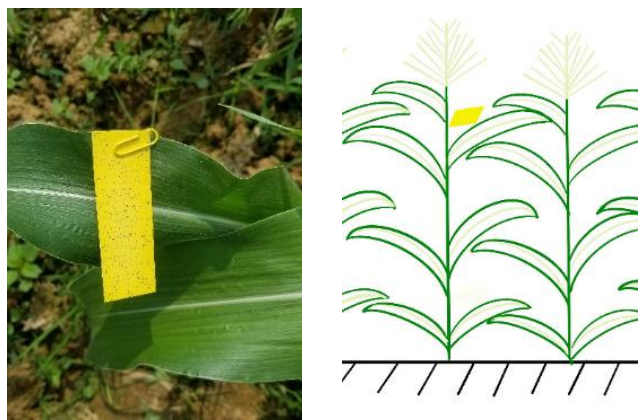

Figure 4 Placement of WSPs and OSPs at each sampling position

\subsubsection{Characterization of pest controls}

The survey and recording of the maize borer were conducted according to pesticide field efficacy test criteria. Based on the incidence of the blank control, three assessments of maize borer were carried out during the tests. The first assessment was conducted prior to the first spray application on June 27. The second and third assessments were conducted on June 30 and July 4. Each assessment was made by sampling five locations on each plot for the larvae. Each plot had 20 plants and each plant was labeled with a hangtag to repeat the survey. This was done according to the National Standard Guidelines for the Field Efficacy Trials-Insecticides Against the Maize Borer in China (GB/T 17980.6-2000).

$$
\begin{aligned}
& \text { Live worm rate }(\%)=\frac{\text { Number of live larvae }}{\text { Number of maize plants }} \\
& \text { Larvae control efficacy }(\%)=\frac{C K_{1}-P T_{1}}{C K_{1}}
\end{aligned}
$$

where, $C K$ is the live worm rate in the blank control and $P T$ is the live worm rate in the treatment.

\subsubsection{Statistical analysis}

The data were analyzed for normality using the Kolmogorov-Smirnov test and for equal variances across the treatments and replicates using Levene's test. Data were compared across using analysis of variance (ANOVA) (SPSS v. 22.0, SPSS Inc., Chicago, IL, USA). Duncan's multiple comparison was used for multiple comparisons, and the significance level was $\alpha=0.05$.

\section{Results}

\subsection{Experimental results of physico-chemical properties experiment}

\subsubsection{Effect of spray adjuvants on DST}

The DST values of the WG solution added 12 kinds of spray adjuvants were measured as shown in Figure 5. The DST value of the WG solution was $40.9 \mathrm{mN} / \mathrm{m}$. It can be seen from the Figure that the DST value of the WG solution decreased by different degrees after adding spray adjuvants. Si1, Si2, and Po2 had the better effect, reducing the surface tension of the original solution by $40.59 \%, 43.77 \%$, and $37.41 \%$, respectively. According to the results of variance analysis, it can be seen that the type of spray adjuvant has a significant influence $(p<0.001)$ on the DST value of the WG solution.

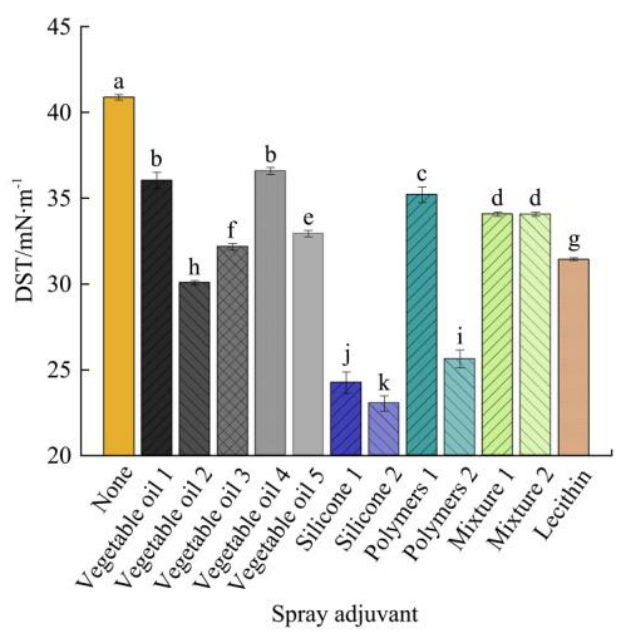

Figure 5 DST of spray adjuvant added to WG

The DST values of the ULV solution with 12 different spray adjuvants were measured (Figure 6). The DST value of the ULV solution was $34.1 \mathrm{mN} / \mathrm{m}$. Compared to the addition of spray adjuvant to the WG solution, the change of spray adjuvant added to the ULV solution was relatively small. The DST of the WG solution increased after most of the adjuvants were added. VO3 had the best effect, reducing the surface tension of the original solution by $12.02 \%$. Analysis of variance (ANOVA) showed that the spray adjuvant had a significant effect $(p<0.001)$ on the DST value of the ULV solution.

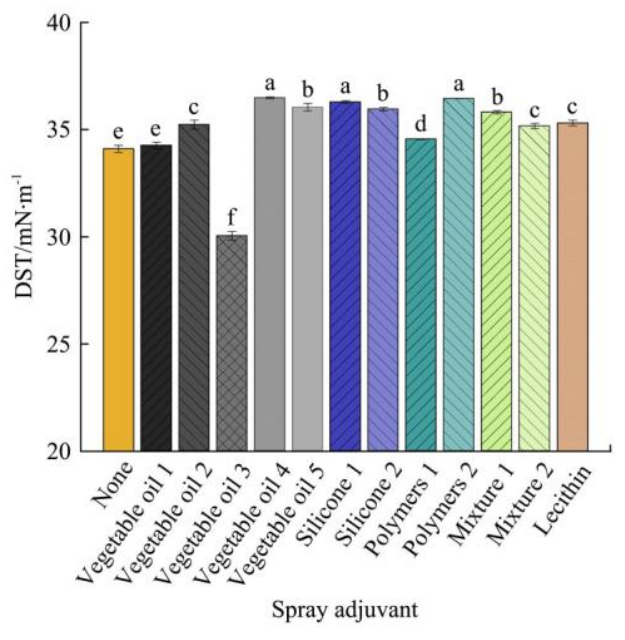

Figure 6 DST of spray adjuvant added to ULV

For the WG solution, silicon produced the greatest reduction of DST due to its extreme wettability. The difference in the ability of the two polymers solutions to decrease DST may be due to the 
manner in which they polymerize. Po2 had a strong ability to decrease DST. The ability of other spray adjuvants to decrease DST was quite different from the above three adjuvants. For the ULV solution, only VO3 had a positive effect on reducing DST. This may be due to the fact that most of the spray adjuvants were developed for water-based pesticides but they were not adaptable for ULVs, which were oil-based pesticide. However, the initial DST of the ULV was lower than that of the WG.

3.1.2 Effect of spray adjuvants on CA

The CA of the WG droplet on the maize leaf decreased gradually over time and tended to be stable at $90 \mathrm{~s}$ (Figure 7). The CA values of WG solution at $0 \mathrm{~s}, 10 \mathrm{~s}, 30 \mathrm{~s}, 60 \mathrm{~s}, 90 \mathrm{~s}$, and $120 \mathrm{~s}$ were $44.71^{\circ}, 31.64^{\circ}, 30.33^{\circ}, 29.19^{\circ}, 26.99^{\circ}$, and $26.30^{\circ}$ respectively. Si1, Si2, and $\mathrm{Po} 2$ had the best effect on allowing the droplets of the WG solution completely moisten and spread on the maize leaves and the $\mathrm{CA}$ was $0^{\circ}$ on leaf contact.

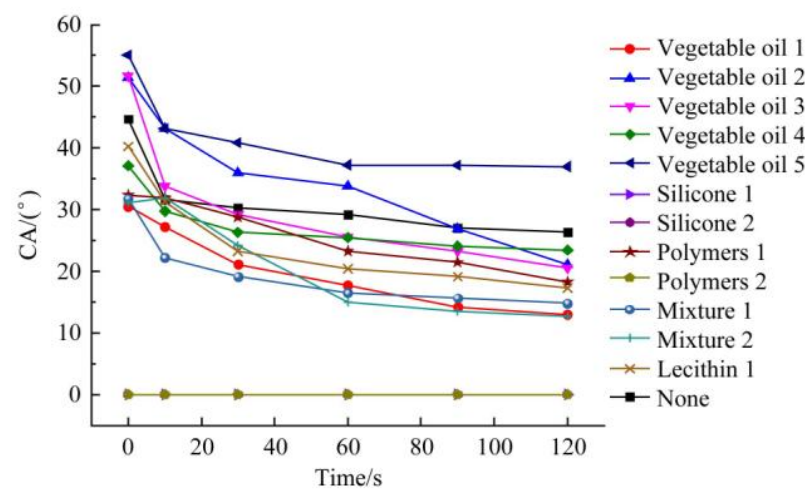

Figure 7 CA of spray adjuvant added to WG solution

The CA of the ULV droplet on the maize leaf decreased gradually with time (Figure 8). ULV solution droplets spread quickly compared with WG solution droplets, and their CA tended to be stable at $30 \mathrm{~s}$. The CA value of the ULV solution at $0 \mathrm{~s}, 10 \mathrm{~s}$, $30 \mathrm{~s}, 60 \mathrm{~s}, 90 \mathrm{~s}$, and $120 \mathrm{~s}$ were $22.21^{\circ}, 14.20^{\circ}, 14.05^{\circ}, 13.79^{\circ}$, $12.51^{\circ}$, and $11.86^{\circ}$, respectively. VO3 was best for allowing the droplets of the ULV solution to completely moisten and spread on the maize leaves. The $\mathrm{CA}$ was $0^{\circ}$ of leaf contact.

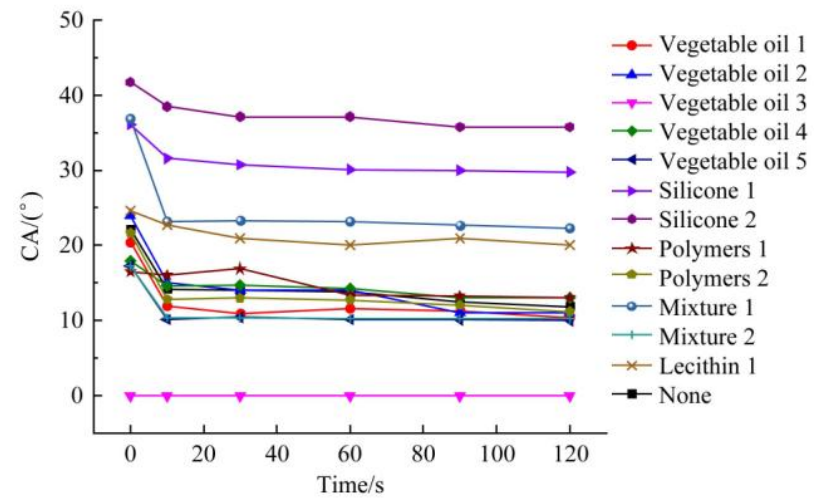

Figure 8 CA of adjuvants added to ULV solution

For the WG solutions, the CA of most treated droplets changed significantly within $0-10 \mathrm{~s}$, and then tended to be stable after $30 \mathrm{~s}$. Similar to the results of the DST tests, the three spray adjuvants with the most significant reduction in DST spread the WG solution completely over the maize leaves. This indicates that $\mathrm{Si} 1, \mathrm{Si}$, and Po2 have good wetting and spreading ability. This allows the drops to spread on the maize leaves and reduces drop loss. For the ULV solution, the CA of most treated droplets changed significantly within $0-10 \mathrm{~s}$, and then tended to be stable. VO3 produced the most significant reduction in DST and allowed the
ULV solution to completely spread over the maize leaves.

In conclusion, the addition of $\mathrm{Si} 1, \mathrm{Si} 2$, and $\mathrm{Po} 2$ to the WG solution and VO3 to the ULV solution significantly improved the physico-chemical properties of the two solutions and improved the wetting and spreading performance of droplets on the leaves. However, some studies show that silicones can have phytotoxicity $^{[26]}$ and the wettability of Po2 was similar to that of the two silicones. Therefore, the WG solution with Po2 and the ULV solution with VO3 were selected for field verification tests.

\subsection{Results of field experiment}

\subsubsection{Droplet deposition and VMD}

Figure 9 shows the droplet deposition and volume median diameter (VMD) of two pesticides at different concentrations and with or without spray adjuvant.It also can be seen that the addition of spray adjuvant and water consumption of the two pesticides increases the droplet deposition, and the increased amplitude differs. The droplet deposition of the WG and ULV at $12 \mathrm{~L} / \mathrm{hm}^{2}$ volume were $0.023 \mu \mathrm{L} / \mathrm{cm}^{2}$ and $0.029 \mu \mathrm{L} / \mathrm{cm}^{2}$. The droplet deposition increased by $104 \%$ after adding spray adjuvant to the WG, and the droplet deposition increased by $239 \%$ after increasing water consumption by $2 \mathrm{~L} / \mathrm{hm}^{2}$ volume. The droplet deposition increased by $24 \%$ after adding spray adjuvant to the ULV, and the droplet deposition increased by $59 \%$ after increasing consumption by $2 \mathrm{~L} / \mathrm{hm}^{2}$ volume. The addition of spray adjuvant $(p=0.024)$ and the water consumption $(p<0.001)$ had significant effects on the droplet deposition of the WG, but not on the droplet deposition of the ULV.

The changes of VMD of the two pesticides after adding spray adjuvant and increasing water consumption were shown in Figure 9. The VMD values of the WG and ULV at $12 \mathrm{~L} / \mathrm{hm}^{2}$ volume were $144 \mu \mathrm{m}$ and $180 \mu \mathrm{m}$, respectively. The VMD increased by $16 \%$ after adding spray adjuvant to the WG, and the droplet deposition increased by $6 \%$ after increasing water consumption by $2 \mathrm{~L} / \mathrm{hm}^{2}$ volume. The droplet deposition was reduced by $3 \%$ after adding spray adjuvant to the ULV, and the droplet deposition increased by $23 \%$ after increasing water by $2 \mathrm{~L} / \mathrm{hm}^{2}$ volume. ANOVA indicated that the addition of spray adjuvant $(p<0.001)$ and water consumption $(p=0.011)$ had significant effects on the droplet deposition of the WG, while water consumption had a significant effect on the droplet deposition of ULV $(p<0.001)$.

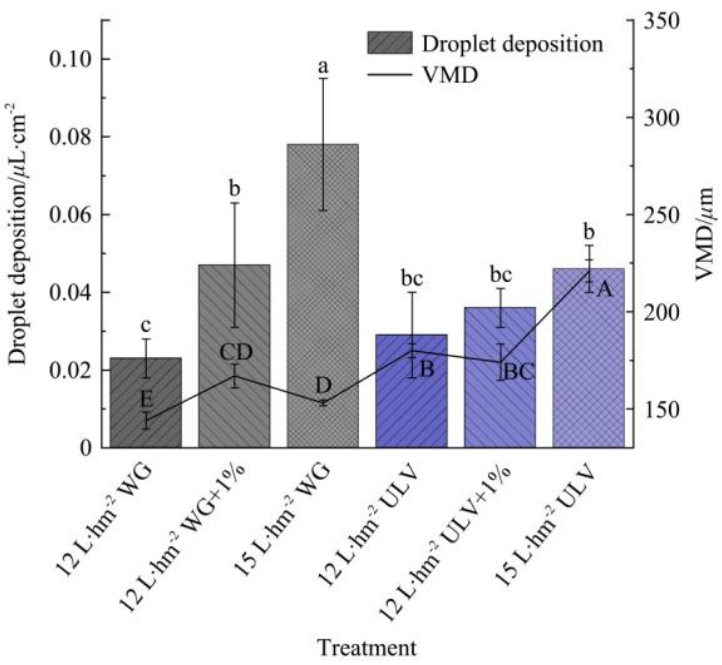

Figure 9 Droplet deposition and VMD by UAV

These results indicate that, for the two pesticides, the addition of spray adjuvant improved droplet deposition. This may have resulted from the increased wettability of the liquid and the larger 
wetting area. The increase in droplet deposition caused by the increase in water consumption was likely caused by the increase in the number of droplets per unit area. The increased droplet deposition will help to improve the control efficacy of the pesticides ${ }^{[27]}$. The difference in the droplet deposition and VMD results of the two pesticides may be caused by the different measurement methods of OSP and WSP, so they were not comparable.

\subsubsection{Control of maize borer}

The control efficacy on maize borer at $3 \mathrm{~d}$ and $7 \mathrm{~d}$ after application by UAV, EAP, and HGC were shown in Figure 10. The best control efficacy was achieved at $15 \mathrm{~L} / \mathrm{hm}^{2}$ volume with the WG by UAV at $3 \mathrm{~d}$ after application ( $76 \%$ control). The best control efficacy ( $88 \%$ control) was achieved at $15 \mathrm{~L} / \mathrm{hm}^{2}$ volume with ULV by UAV at $7 \mathrm{~d}$ after application.

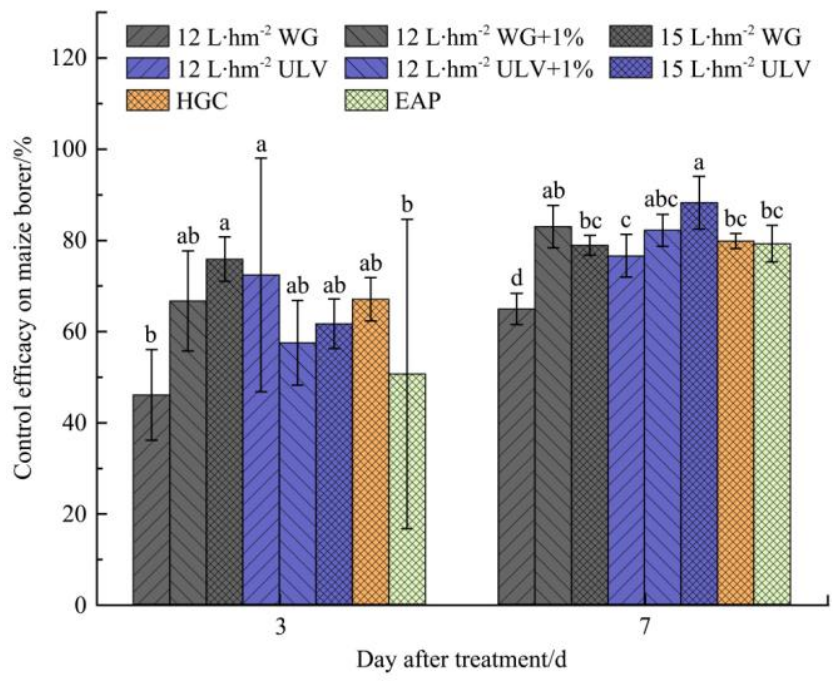

Figure 10 Control efficacy on maize borer at $3 \mathrm{~d}$ and $7 \mathrm{~d}$ after application

At $3 \mathrm{~d}$ after application, the addition of spray adjuvant $(P=$ $0.020)$ and water consumption $(p=0.001)$ significantly improved the control efficacy of the WG solution by $46 \%$ and $65 \%$, respectively. However, the effects on the ULV solution were not significant. The treatments by UAVs were not significantly different from the control provided by HGC with WG. The treatments using UAVs at $15 \mathrm{~L} / \mathrm{hm}^{2}$ volume with WG $(p=0.008)$ and $12 \mathrm{~L} / \mathrm{hm}^{2}$ volume with ULV $(p=0.021)$ were significantly different from the control using EAP with WG.

Addition of spray adjuvant $(p<0.001)$ and water consumption $(p<0.001)$ to the WG solution significantly improved the control efficacy at $7 \mathrm{~d}$ after treatment with increased control efficacy of $27 \%$ and $22 \%$, respectively. Consumption had a significant effect on the control efficacy of ULV with increased control efficacy of 14\%. The treatments using UAVs at $12 \mathrm{~L} / \mathrm{hm}^{2}$ volume with WG were worse than those of control using HGC and EZP with WG, and they were significantly different. This indicates that most of the control efficacy of the UAV sprayer meets basic field control requirements.

The results indicated that the addition of spray adjuvant significantly improved the control efficacy of the WG solution, while the effect was not significant for the ULV solution. This may have been caused by the good wettability of the ULV solution itself. Increasing the water consumption can also improve the control efficacy of the two pesticide formulations, but the difference was not significant compared with adding spray adjuvant. The purpose of reducing water consumption can therefore be achieved by adding spray adjuvant.

In conclusion, the addition of spray adjuvant (Po2) to the WG formulation significantly improved the droplet deposition and VMD, thereby reducing drift loss and causing more droplets to deposit on the target. The addition of spray adjuvant (Po2) to WG significantly improved the control efficacy, while the control efficacy with the addition of spray adjuvant and the control efficacy with the increase of water consumption were similar. The ideal control efficacy can be achieved by using the WG at $12 \mathrm{~L} / \mathrm{hm}^{2}$ volume in combination with a spray adjuvant. The water consumption can be reduced by $20 \%$ by adding spray adjuvant and this mix will meet field insect control requirements.

\section{Discussion}

The results demonstrate the wettability differences (represented by the DST and CA) of several spray adjuvants and the importance of pesticide spray wettability (achieved by adding adjuvant) on the quality of the spray distribution and control efficacy.

The DSTs of the silicone spray adjuvants (Si1 and Si2) to WG were significantly lower than that of other treatments, which was consistent with the results of Stevens on the super ductility of organosilicon $^{[28]}$. The hyperbranched polymer spray adjuvant (Po2) had a strong effect on reducing tension compared with vegetable oil, mixture, and others. Zhang et al. ${ }^{[2]}$ found that hyperbranched polymers can improve the wetting and spreading performance of solutions. Although other spray adjuvants can also play a role in reducing DST, their effects, compared with the above three spray adjuvants, were still uncertain. Depending on the composition, different vegetable oil spray adjuvants have different abilities to decrease the DST.

Both silicone and hyperbranched polymer spray adjuvants can decrease the CA of WG on maize leaves to $0^{\circ}$. This is because spray adjuvants decrease the DST of the solution to below the critical surface tension of maize leaves, causing the droplets to fully wet and spread out on the leaves. Wang et al. showed that the DST of the solution was lower than that of the critical surface tension of plant leaves and the droplets could wet and spread out on the leaves ${ }^{[30]}$. The lower the DST and CA of the droplet, the easier it is to moisten and spread on the target, and the greater the amount of the active component of the liquid will be absorbed by the target ${ }^{[31]}$. Polymer 2 provides similar wetting and spreading ability of the liquid as do the silicone spray adjuvants. To avoid the possible phytotoxicity of the silicone spray adjuvants, Po2 was selected from 12 spray adjuvants for combination with WGs for maize treatment.

Except for the VO3 spray adjuvant, other spray adjuvants did not decrease the DST of the ULV. This may be because most of the spray adjuvants were developed for water-based pesticides and were not adaptable for ULV, an oil-based formulation. Oil is denser than water. Maize leaves have more hair on their surfaces and are more hydrophobic ${ }^{[32]}$, so an oil-based pesticide can be spread more quickly and easily on the surface of maize leaves. Because of this, VO3 was selected for combination with the ULV solution to treat maize leaves.

Adding spray adjuvant to WGs significantly increased droplet size and droplet deposition. With the increased wettability of the pesticide formulation, the spread area of a single droplet will be larger and lead to larger droplet deposition per unit area ${ }^{[33]}$. However, adding spray adjuvant to the ULV did not significantly affect the droplet deposition, so it did not affect droplet wetting and spreading performance. 
The addition of spray adjuvant to WGs significantly improved insect control efficacy, but the difference was not significant compared with efficacy improvement caused by increased water consumption. This is because increased droplet deposition also led to improved control efficacy. The addition of spray adjuvant and the increase of water consumption in the ULV did not influence the results of droplet deposition, so the differences in control efficacy were not significant.

Even though the UAV sprayer applied a significantly lower water volume and had a lower percentage surface area coverage, the control efficacy of the UAV was similar to that of the EAP sprayer and HGC sprayer. This is because the droplet size produced by the UAV nozzle was significantly smaller than the droplets produced by the EAP sprayer and HGC sprayer. The number of spray droplets per area has a large impact on the level of pest control. In pesticide applications, it is not necessary to use a large amount of solution, an optimal number of spray deposits can achieve good efficacy, particularly for systemic pesticides ${ }^{[27]}$.

This study demonstrated that the wettability of droplets during UAV operation can be improved by adding an appropriate spray adjuvant. This will increase droplet deposition and improve insect control efficacy. Droplet deposition and control efficacy can be achieved by adding spray adjuvant to a conventional water-based pesticide or by increasing the water consumption, which improves the operational efficiency of the UAV. The ULV formulation has excellent wettability and control efficacy without the addition of a spray adjuvant. Future studies should assess whether the addition of adjuvants and special pesticides for agricultural aviation can reduce evaporation and drift.

\section{Conclusions}

The aims of this study were to determine the effects of different aviation spray adjuvants on formulation wettability and spay efficacy in maize borers controlling. The effect of the optimized adjuvants on maize insecticide formulations was verified by the results of droplet deposition and the control efficacy on maize borers. The results were as follows:

1) Spray adjuvants $\mathrm{Si} 1, \mathrm{Si} 2$, and $\mathrm{Po} 2$ were most effective on improving the wettability of the WG. For the ULV solution, the best effect was provided by VO3. To avoid the potential effects of silicon phytotoxicity, Po2 was added to the WG and VO3 was added to the ULV solution for advanced testing.

2) Field experiments demonstrated that Po2 significantly improved droplet deposition and insect control efficacy. The spray adjuvants Po 2 can reduce the water consumption by $20 \%$ and achieve the $83 \%$ control efficacy on maize borer. The effect of VO3 on improving the droplet deposition and control efficacy of the ULV solution was not significant.

\section{Acknowledgements}

We thank the National Key Research and Development Project of China "Comprehensive technology research and development of chemical fertilizer and pesticide application reduction and efficiency enhancement" (Grant No. 2018YFD0200702), Guangdong Basic and Applied Basic Research Foundation (Grant No. 2020A1515110214), National Natural Science Foundation of China (Grant No. 31901401), and Project of Rural Revitalization Strategy in Guangdong Province (Grant No. 2020KJ261) for funding. We also thank the anonymous reviewers for their critical comments and suggestions for improving the manuscript. We thank LetPub (www.letpub.com) for its linguistic assistance during the preparation of this manuscript.

\section{[References]}

[1] Wang Z Y, He K L, Zhang F, Lu X, Babendreier D. Mass rearing and release of Trichogramma for biological control of insect pests of corn in China. Biol Control, 2014; 68: 136-144.

[2] Wang G B, Lan Y B, Qi H X, Chen P C, Hewitt A, Han Y X. Field evaluation of an unmanned aerial vehicle (UAV) sprayer: effect of spray volume on deposition and the control of pests and disease in wheat. Pest Manag Sci, 2019; 75(6): 1546-1555.

[3] Zheng Y J, Yang S H, Zhao C J, Chen L P, Lan Y B, Yu T. Modelling operation parameters of UAV on spray effects at different growth stages of corns. Int J Agric \& Biol Eng, 2017; 10(3): 57-66.

[4] Omar Z, Idris N, Rahim M Z. Preliminary design of aerial spraying system for microlight aircraft. Journal of Physics Conference Series, 2017; 914(1): 012003. doi:10.1088/1742-6596/914/1/012003

[5] García-Santos G, Feola G, Nuyttens D, Diaz Z. Drift from the use of hand-held knapsack pesticide sprayers in Boyacá (Colombian Andes). J Agr Food Chem, 2016; 64(20): 3990-3998.

[6] Cao L D, Cao C, Wang Y, Li X H, Zhou Z L, Li F M, et al. Visual determination of potential dermal and inhalation exposure using Allura red as an environmentally friendly pesticide surrogate. Acs Sustain Chem Eng, 2017; 5(5): 3882-3889.

[7] Zang Y, Gu X Y, Zhou Z Y, Luo X W, Zang Y, Qi X Y, et al. Review of tensairity and its applications in agricultural aviation. Int J Agric \& Biol Eng, 2016; 9(3): 1-14.

[8] Rasmussen J, Nielsen J, Garcia-Ruiz F, Christensen S, Streibig J C, Lotz B Potential uses of small unmanned aircraft systems (UAS) in weed research. Weed Res, 2013; 53(4): 242-248.

[9] Zang Y, Zang Y, Zhou Z Y, Gu X Y, Jiang R, Kong L X, et al. Design and anti-sway performance testing of pesticide tanks in spraying UAVs. Int J Agric \& Biol Eng, 2019; 12(1): 10-16.

[10] Chen S D, Lan Y B, Li J Y, Zhou Z Y, Liu A M, Mao Y D. Effect of wind field below unmanned helicopter on droplet deposition distribution of aerial spraying. Int J Agric \& Biol Eng, 2017; 10(3): 67-77.

[11] Appah S, Jia W D, Ou M X, Wang P, Asante E A. Analysis of potential impaction and phytotoxicity of surfactant-plant surface interaction in pesticide application. Crop Prot, 2020; 127: doi: 104961. 10.1016/ j.cropro.2019.104961

[12] De Assuncao H H T, Campos SFB, Sousa L A, Lemes E M, Zandonadi C H S, da Cunha J P A R. Adjuvants plus phytosanitary products and the effects on the physical-chemical properties of the spray liquids. Biosci J, 2019; 35(6): 1878-1885.

[13] Calou V B C, Teixeira A D S, Moreira L C J, Da Rocha Neto O C, Da Silva J A. Estimation of maize biomass using unmanned aerial vehicles. Engenharia Agrícola, 2019; 39(6): 744-752.

[14] Zhang M N, Zhou J F, Sudduth KA, Kitchen NR. Estimation of maize yield and effects of variable-rate nitrogen application using UAV-based RGB imagery. Biosyst Eng., 2020; 189: 24-35.

[15] Zhang L Y, Niu Y X, Zhang HH, Han WT, Li G, Tang J D, et al. Maize canopy temperature extracted from UAV thermal and RGB imagery and its application in water stress monitoring. Front Plant Sci., 2019; 10: 1270.

[16] Xiao Q G, Xin F, Lou Z X, Zhou T T, Wang G B, Han X Q, et al. Effect of aviation spray adjuvants on defoliant droplet deposition and cotton defoliation efficacy sprayed by unmanned aerial vehicles. Agronomy-Basel, 2019; 9: 2175.

[17] Meng Y H, Lan Y B, Mei G Y, Guo Y W, Song J L, Wang Z G. Effect of aerial spray adjuvant applying on the efficiency of small unmanned aerial vehicle for wheat aphids control. Int J Agr Biol Eng, 2018; 11(5): 46-53.

[18] Klein R N, Golus J A, Nelms K L. The effect of adjuvants, pesticide formulation, and spray nozzle tips on spray droplet size. J Astm Int., 2009; 6(6): 1-7.

[19] Wang X N, He X K, Song J L, Wang Z C, Wang C L, Wang S L, et al. Drift potential of UAV with adjuvants in aerial applications. Int J Agric \& Biol Eng, 2018; 11(5): 54-58.

[20] Zhu Y Q, Yu C X, Li Y, Zhu Q Q, Zhou L, Cao C, et al. Research on the changes in wettability of rice (Oryza sativa.) leaf surfaces at different development stages using the OWRK method. Pest Manag Sci, 2014; 70(3): 462-469.

[21] Castro E B, Carbonari C A, Velini E D, Gomes G L G C, Belapart D. Influence of adjuvants on the surface tension, deposition and effectiveness 
of herbicides on fleabane plants. Planta Daninha, 2018. (In press).

[22] Gao Y, Lu J J, Zhang P J, Shi G C, Li Y, Zhao J Y, et al. Wetting and adhesion behavior on apple tree leaf surface by adding different surfactants. Colloids Surf. B, 2020; 187: 110602.

[23] Fritz B K, Hoffmann W C, Martin D E, Thomson S J. Aerial application methods for increasing spray deposition on wheat heads. Appl Eng Agric, 2007; 23(6): 709-715.

[24] Zhu H P, Salyani M, Fox R D. A portable scanning system for evaluation of spray deposit distribution. Comput Electron Agr, 2011; 76(1): 38-43.

[25] Lan Y, Hoffmann W C, Fritz B K, Martin D E, Lopez J D. Spraydrift mitigation with spray mix adjuvants. Appl Eng Agric, 2008; 24(1): 5-10.

[26] Sun J S, Policello G A, Paccione M A. Determination of organosilicone surfactant phytotoxicity for selected vegetable species. Pesticide Formulations and Application Systems, 2003; 23: 77-84.

[27] Yuan H Z, Wang G B. Effects of droplet size and deposition density on filed efficacy of pesticides. Plant Protection, 2015; 41(6): 9-16. (in Chinese)

[28] Stevens P J G. Organosilicone surfactants as adjuvants for agrochemicals. Pest Management Science, 2010; 38: 103-122.
[29] Zhang Z J, Shen L W, Hu W X, Mi Y Z, Yuan H K, Kuang J Z, et al. Treatment of oily wastewater using a hyperbranched poly (amido amine) demulsifier with 1,4-phenylene diamine as central core. Chemistry Select, 2020; 32(5): 9980-9988.

[30] Wang S, Pan W X, Gao N, Zhang S S, Zhang H. Effect of adjuvants on wetting performance and efficacy of topramezone $30 \%$ SC. Agrochemicals, 2020; 59(6): 413-417. (in Chinese)

[31] Zhang C H, Ma Y, Du F P. Research progress on the wetting and deposition behaviors of pesticide droplet on target surfaces with the addition of surfactants. Chinese Journal of Pesticide Science, 2019; 21(Z1): 5-6. (in Chinese)

[32] Mc Tanure M, da Costa L M, Huiz H A, Fernandes R B A, Cecon P R, Pereira J D, et al. Soil water retention, physiological characteristics, and growth of maize plants in response to biochar application to soil. Soil Till Res, 2019; 192: 164-173.

[33] Hui F, Zhang Z Q, Xiao S P, Liu Y F. Influence of leaf surface wettability on droplet deposition effect of rape leaves and their correlation. Journal of Agriculture and Food Research, 2019; 1: 100011. doi: 10.1016/j.jafr.2019.100011 